OPEN ACCESS

Edited by:

Zhao Wang,

University of Electronic Science and Technology of China, China

Reviewed by:

Atsushi Sakamoto,

CVPath Institute, United States Yuanli Chen,

Hefei University of Technology, China Meixiu Jiang,

Nanchang University, China

*Correspondence:

Zhongqun Wang wangtsmc@126.com

Specialty section:

This article was submitted to Cardiovascular Imaging, a section of the journal

Frontiers in Cardiovascular Medicine

Received: 29 May 2021

Accepted: 30 August 2021

Published: 29 September 2021

Citation:

Zhang L, Li L, Feng G, Fan T, Jiang H and Wang $Z$ (2021) Advances in CT Techniques in Vascular Calcification. Front. Cardiovasc. Med. 8:716822.

doi: 10.3389/fcrm.2021.716822

\section{Advances in CT Techniques in Vascular Calcification}

\author{
Lijie Zhang ${ }^{1}$, Lihua Li $^{2}$, Guoquan Feng ${ }^{3}$, Tingpan Fan ${ }^{1}$, Han Jiang ${ }^{1}$ and Zhongqun Wang ${ }^{1 *}$ \\ ${ }^{1}$ Department of Cardiology, Affiliated Hospital of Jiangsu University, Zhenjiang, China, ${ }^{2}$ Department of Pathology, Affiliated \\ Hospital of Jiangsu University, Zhenjiang, China, ${ }^{3}$ Department of Radiology, Affiliated Hospital of Jiangsu University, \\ Zhenjiang, China
}

Vascular calcification, a common pathological phenomenon in atherosclerosis, diabetes, hypertension, and other diseases, increases the incidence and mortality of cardiovascular diseases. Therefore, the prevention and detection of vascular calcification play an important role. At present, various techniques have been applied to the analysis of vascular calcification, but clinical examination mainly depends on non-invasive and invasive imaging methods to detect and quantify. Computed tomography (CT), as a commonly used clinical examination method, can analyze vascular calcification. In recent years, with the development of technology, in addition to traditional CT, some emerging types of CT, such as dual-energy CT and micro CT, have emerged for vascular imaging and providing anatomical information for calcification. This review focuses on the latest application of various CT techniques in vascular calcification.

Keywords: vascular calcification, calcification score, micro CT, dual energy CT, multi-slice spiral CT

\section{INTRODUCTION}

In hypertension, diabetes, atherosclerosis, and other diseases, vascular calcification, which severely affects human health, must not be ignored (1). Currently, there are many quantitative methods to calculate a vascular calcification level score. The most commonly used process is the Agatston (2), in which lesions with CT value $\geq 130 \mathrm{Hu}$ and area $\geq 1 \mathrm{~mm}^{2}$ (3) are defined as calcification. It is worth noting that the Agatston score is for the assessment of calcification in the coronary artery. As a clinically accessible imaging technique, $\mathrm{CT}$ is the most advanced non-invasive tool for detecting coronary calcification (4). CT can quickly and easily image and analyze the calcification. Bradley et al. (5) divided calcification into three characteristics: perimeter, length, and morphology, and the score accorded each feature. Multiple studies (6) have verified the accuracy and importance of calcification score derived by CT. In this review, recent development in various CT techniques in evaluating vascular calcification is stated. Moreover, we discuss the application and future development of CT in vascular calcification.

\section{THE SIGNIFICANCE OF VASCULAR CALCIFICATION ASSESSED BY CT, ESPECIALLY IN THE CORONARY ARTERY}

$\mathrm{CT}$ is a non-invasive imaging method for evaluating and analyzing vascular calcification. It is considered the gold standard for analyzing and quantifying vascular calcification $(7,8)$. Many studies have shown that the coronary calcium score is an essential and reliable predictor as levels of calcium increase with the morbidity and mortality of cardiovascular disease in patients (9-11). In a feasibility study, patients with a calcium score over 400 had a high risk of cardiovascular disease, 
while those who scored 1 to 400 were at twice the risk of cardiovascular disease as those with a zero score (12). Rennenberg et al. analyzed the results of 30 different studies. They found a three to four times higher risk of incidence and mortality of cardiovascular disease in patients with vascular calcification (13).

Coronary calcium scoring is useful for a wide range of factors such as age and risk. It can assist risk stratification for clinical cardiovascular events $(14,15)$. Furthermore, the coronary artery calcium score (CACS) helps improve risk prediction in intermediate-risk groups when combined with other traditional clinical risk classification methods (16). This was confirmed by Tamar et al. (17). They found a greater redistribution of risk for middle-grade patients. This suggests that middlerisk patients may be more appropriate for coronary calcium scores. CACS was verified as optimizing the risk classification of cardiovascular events. It has significantly improved the accuracy of cardiovascular risk stratification. More importantly, CACS can help some patients at risk of cardiovascular disease be treated with statins at a secondary prevention level (18).

As a relatively inexpensive non-invasive imaging method (19), coronary computed tomography (CCTA) has become a vital examination method of patients with coronary disease in the clinical diagnosis and treatment plans due to its advantages of high spatial resolution, high sensitivity (95-99\%), and high negative predictive value (97-99\%) (20). The resolution of currently available CCTA is $0.5-1 \mathrm{~mm}$, and CCTA is widely used to prevent and examine coronary heart disease (21). As a non-invasive examination method, compared with invasive coronary angiography, the visualization of blood vessels is the main advantage of CCTA (22). It provides information about coronary arteries' structure and information about the shape and composition of vascular plaques. Furthermore, the severity of coronary plaques estimated by imaging is consistent with intravascular ultrasound (IVUS) (23). However, it cannot detect minute elements such as macrophage accumulation and prominent plaque characteristics (24).

Using the traditional CT scanner, coronary artery calcium (CAC) is defined as (3) a lesion above the threshold of 130 sound field units with an area $\geq 1 \mathrm{~mm}^{2}$. Studies have shown that (25) for lesions with calcification score $\geq 400$, the sensitivity and specificity of conventional CT in the diagnosis of coronary heart disease are lower than those of CCTA technology. Furthermore, as CCTA provides less information when the calcification score is high, severely calcified plaques cause beam hardening and blooming artifacts, resulting in inaccurate diagnoses of coronary artery stenosis (26). Skinner et al. (27) recommended invasive coronary angiography for patients with a CAC score $>400$. It has been proved that (28) adding the transluminal attenuation gradient of a transverse optical lumen to CCTA can improve the diagnostic accuracy of CCTA. Experiments show that (29) a higher heart rate will affect the repeatability of CCTA plaque measurement. Higher heart rates will produce motion artifacts, leading to poor plaque image quality. Some strategies have been developed to improve CCTA diagnostic performance in calcified plaque recognition $(30,31)$. These methods include using image post-processing methods and iterative reconstruction (IR) algorithms to suppress the influence of severe calcification on coronary artery lumen evaluation. Li et al. (32) found that the blooming removal algorithm significantly reduced blooming artifacts caused by calcified plaque, reduced the occurrence of false-positive coronary artery lesions, and improved CCTA diagnostic accuracy. It is worth noting that a study found that (33) the relationship between calcium volume and density and subsequent clinical diseases varies. Coronary artery calcification volume is positively correlated with both coronary heart disease and cardiovascular disease, while calcification density is inversely proportional to both. Several other studies have supported this view, concluding that stabilization has a greater density of calcification plaques than acute coronary heart disease (34-37). These results suggest that the density of calcification plaques may be protective and that high densities of calcification plaques are associated with stability. The mechanism by which this phenomenon occurs is currently unknown (38), and further investigation is needed.

CAC degree is closely associated with age, sex, and other factors, and the extent and prevalence of calcification increase with age (39). CCTA can detect CAC before patient symptoms appear, thus shortening hospital stay and saving costs (40). However, its main disadvantages are the need for iodized contrast media and radiation exposure, and consequently, there may be a potential risk of radiation-related malignant tumors (41).

\section{WHAT ARE THE INADEQUACIES AND WEAK POINTS OF CALCIFICATION DETECTION BY EXISTING CT?}

Depending on the vessel site involved, vascular calcification can be classified into medial and intimal calcification (42). As the spatial resolution of normal CT is not ideal, distinguishing between intimal and medial calcification is challenging. Normal CT does not detect microcalcification effectively (8). Additionally, with the widespread use of CT in vascular calcification imaging, radiation exposure is a significant concern. Consequently, reducing radiation exposure while maintaining image quality has become the focus of subsequent technical improvements (43). Currently, four techniques are used to optimize the radiation dose in multi-slice spiral CT (MSCT) arterial imaging: an ECG control tube technique, automatic exposure technique, tube voltage adjustment, and extensive pitch selection technique. It has also been proved that (44) 640-slice CT technology can reduce the radiation dose using wide-area detector technology. However, the expensive CT equipment and high detection cost also limit its wider use (45).

Imaging by $\mathrm{CT}$, high-risk plaques include napkin ring signs, spot calcification, low attenuation plaques, and positive remodeling. The density of the napkin ring sign area is characterized by a high outer and low inner density (46), spot calcification of $<3 \mathrm{~mm}$ (47), low attenuation feature patches <150 HU (48), and positive remodeling RI $>1.1$ (47). If two of these are satisfied, it is defined as high-risk plaque (47). High-risk plaques are undesirable characteristics and can significantly increase the risk of cardiovascular disease. 
Contrastingly, significant calcification can stabilizes the plaque and rarely rupture, reflecting the disease stability (49).

\section{CURRENT CLINICALLY AVAILABLE CT TECHNIQUES \\ MSCT}

With the development of medical technology, MSCT has been constantly updated and improved, expanding from its previous 16-slice system to 128-, 256-, 320-, and 640-slice, or more (21). The development of multilayer spiral CT significantly reduces the patient's breath-holding time. At the same time, the improvement in temporal resolution reduces cardiac motion artifacts (50). After long-term clinical application, MSCT is a non-invasive, highly safe, operationally simple technique that can analyze coronary artery calcification and accurately assess the severity of coronary artery stenosis and plaque composition (21). This provides a variety of information for follow-up treatments.

With the gradual improvement in spatial resolution, MSCT allows the complete imaging analysis of minute calcification, and repeated examinations can be performed (21). MSCT is not only fast, simple, and non-invasive with high diagnostic accuracy but also uses many post-processing technologies (51), including volume rendering (VR), maximum intensity projection (MIP), multiplanar reconstruction (MPR), and curved planar reconstruction (CPR). However, these post-processing techniques also have their shortcomings. The role of each postprocessing technique is different; for example, VR is beneficial to the observation of the overall arterial anatomy, while CPR is useful in plaque estimation (51). Many post-processing techniques are often used in combination to avoid misdiagnosis or no diagnosis at all.

Over the past decade, MSCT techniques can accurately detect and quantify the degree of arterial calcification. It has been used to monitor the progress of vascular calcification and evaluate and compare the effectiveness of various treatment regimens (45), including assessing the role of vitamin D in CAC (52). Medical staff can integrate CT into the decision-making process and improve work efficiency. Importantly, MSCT has been used to examine vascular calcification, distinguish between patients with different cardiovascular disease incidences and mortality risks, and conduct timely clinical interventions to improve patient disease management and improve patient care.

The dual-source CT (DSCT) is a new technology developed based on 64-slice spiral CT. DSCT with two x-ray tubes and two detectors and the systems can work simultaneously (53). DSCT improves temporal resolution compared to single-source CT, which allows for imaging at higher heart rates. Table $\mathbf{1}$ compares the main parameters of the first-, second-, and third-generation DSCT $(54,55)$. With the introduction of third-generation DSCT, its focus is smaller than that of previous generations. Even small anatomy can be displayed with a superior image quality, compared to the previous CT (56). Recently, it was found that the third-generation DSCT can be combined with tin filtering to reduce the radiation dose during calcification image acquisition $(57,58)$. In another study, Manta et al. (59) used the thirdgeneration DSCT to image calcification in mice, and the scanning time was only $40 \mathrm{~s}$. The total calcium content of detected calcified aortic plaques was as low as $0.71 \mu \mathrm{g} \mathrm{Ca}^{2+} / \mathrm{mg}$, proving the feasibility of imaging human-calcified plaques using the CT system. Moreover, the experimental results of Philip et al. showed that the radiation dose during CCTA, using third-generation DSCT, was reduced, and image quality was better than with the second-generation DSCT. They propose that the latest thirdgeneration DSCT CCTA can be performed on patients with a radiation dose of $<1 \mathrm{mSv}$ (60).

\section{Dual-Energy CT}

Dual-energy CT (DECT), also called (61) spectral CT, can image the exact location using two different $\mathrm{kVp}$ so that two other datasets are obtained (62). Presently, DECT uses six methods and techniques (63), namely, dual-source DECT, single-source helical DECT, single-source twin-beam DECT, single-source sequential DECT, single-source rapid switching DECT, and dual-layer DECT. Dual-source DECT has a two-source CT system. Each $\mathrm{X}$-ray tube produces a different X-ray energy spectrum. Singlesource helical DECT is performed with two spiral scans under different $\mathrm{kVp}$ conditions, and single-source twin-beam DECT features the use of split-filter technology. Single-source sequential DECT data are obtained twice with two different $\mathrm{kVp}$, singlesource rapid switching DECT features an immediate change in the tube voltage between 80 and $140 \mathrm{kVp}$, and dual-layer DECT has a unique dual-layer energy-resolving detector (63).

TABLE 1 | Comparison of first-, second-, and third-generation dual-source CT.

\begin{tabular}{lccc}
\hline Main parameter & First generation & Second generation & Third generation \\
\hline Minimum frame rotation time (s) & 0.33 & 0.28 & 75 \\
Time resolution (ms) & 83 & 45.8 & 66 \\
Max scan speed (cm/s) & 20.0 & 128 & 73.7 \\
Maximum number of image layers (layer) & 64 & 70.80 .100 .120 .140 & 96 \\
Optional kV value (KV) & 80.100 .120 .140 & 33 & 50 \\
Imaging vision (cm) & 26 & $2 \times 100$ & $2 \times 120$ \\
Ball tube max. power (kW) & $2 \times 80$ & 34.8 & 52.5
\end{tabular}


DECT features two different grades of images which can be obtained by one scan and can distinguish between the calcified and non-calcified plaque as well as show the stenosis degree of coronary artery lumen (64). Another advantage of DECT (65) is that it simplifies the workflow. Its data apply to all imaged patients; there is no need to select patients before scanning. DECT can remove artifacts and clearly show the components that produce artifacts such as coronary calcification and metals (66). DECT application technology (67) includes virtual monochromatic imaging (VMI) and virtual non-contrast (VNC) reconstruction. VMI is the synthetic image at a specific single energy level from two spectral $\mathrm{x}$-ray projections, and VNC removes some imaging components (68). Using VMI to reduce the contrast agent dose is considered one of the most promising DECT applications (67). Additionally, VMI has the advantages of improving image contrast (69) and reducing artifacts (70), consequently improving the overall image quality and diagnostic performance. However, VMI has several limitations in reducing artifacts (71); the effect is best at high keV, but this also suppresses the iodine contrast. In addition, the removal of metal artifacts at high $\mathrm{keV}$ depends on the properties of the metal itself. VNC can be used to obtain the calcium score of coronary arteries (72). Compared with using calcium score acquisition alone, the radiation dose was reduced, but the displayed calcification was smaller than the actual calcification (73). Therefore, further research is still needed to determine the specific deviation of the VNC from reality (68). In a phantom study, researchers used a plaque similar to human vascular calcification and demonstrated strong agreement between the calcium integration calculated by DECT and the conventional mono-energy CT (74). It takes some time to use these techniques in fast-paced clinical work, which makes it challenging to get the maximum advantages of DECT (75).

DECT uses material decomposition to reduce the calcified components of plaque, thereby improving the visualization of the optical cavity (68). It also enhances plaque visualization to enable the accurate assessment of high-risk plaque features (76). DECT achieves a high-pitch spiral acquisition protocol of 3.0 and higher, shortens the scanning time, and thus reduces the effective radiation dose (77). DECT has a unique acquisition method called high-pitch spiral acquisition. It has a pair of dual-energy detectors. It only uses a quarter of the rotation time to obtain an image, thus providing a higher time resolution (78). According to a study (79), the imaging of ultrasmall superparamagnetic iron oxide by DECT may be helpful to visualize and quantify the accumulation of macrophages in plaque. It is expected that this technique will become a new technique in coronary plaque imaging. Ultrasmall superparamagnetic iron oxide is a negatively charged contrast agent that can stay in the circulatory system for a long time (80).

Logically, the total radiation dose of DECT is twice that of traditional scanning because it needs to obtain data at two different energy levels. However, DECT uses some methods to divide the total radiation dose into high- and low-energy components so that the total dose is no higher than in conventional scanning $(81,82)$. DECT can subtract calcified plaques from the images, which may improve the assessment of the vascular system, especially with severely calcified plaques (71). Similarly, Domenic et al. (83) evaluated the DECT scanning calcium subtraction algorithm and its influence on the intracavity visualization of patients with severely calcified coronary arteries. They found that compared with the standard linear mixed nonsubtraction image, the image with calcium subtraction provided better visibility of the coronary artery lumen and improved the reliability of diagnosis without affecting image quality and contrast noise. However, Michael et al. (84) found that DECT is not effective in evaluating the integrity of blood vessels and the plaque subtraction results are biased. Consequently, additional research is also needed to assess the role of this technique under specific clinical conditions.

In conclusion, further research is still needed in using DECT to identify coronary plaque and evaluate its diagnostic performance and potential clinical value.

\section{CURRENT AVAILABLE NON-CLINICAL NEW CT TECHNIQUES}

\section{Micro-CT}

Micro-CT has received wide attention as a newly developed imaging method for examining vascular calcification. Microcalcification in the fibrous cap destroys plaque stability by promoting rupture and is not easily detected by the twodimensional histological method (85). A microcalcification size of only between 5 and $65 \mu \mathrm{m}$ is sufficient to make the plaque unstable (86). However, ordinary CT cannot effectively detect microcalcification due to spatial resolution limitations. The spatial resolution of micro-CT can reach 1-10 $\mu \mathrm{m}$ (87), which can distinguish and quantify microscopic and macroscopic calcification. Moreover, micro-CT can detect microcalcifications in blood vessels which are normally difficult to find. In animal research, histological methods have been used for calcification analysis, but they have some shortcomings, such as the inability to check for complete vascular calcification. However, using micro-CT surmounts these limitations and calcification can be visualized and quantified three-dimensionally (88). Micro-CT can quantify calcification volume and calcification load, and there was no experimental deviation in the localization and distribution of calcification (88). Calcification load has a strong correlation with the calcification score. The volume represents the spatial size of the calcification plaque and the unit of calcification volume in $\mathrm{mm}^{3}$ (89). Although micro-CT is reliable in detecting calcified plaque, it cannot effectively visualize the calcified internal structure, limiting the imaging of small structures such as calcified cell recesses and cell cracks (90). As previously stated, the disadvantages of micro-CT (91) include a long acquisition time, the need for deep anesthesia, poor soft tissue contrast, and high radiation dose, and the radiation doses can reach $760 \mathrm{mGy}$ per scan. However, the CCTA radiation dose is usually around $100-450 \mathrm{mGy}$ (92).

Non-destructive 3D micro-CT has been used in some studies on preclinical vascular calcification. Current CT 3D imaging technology can completely reconstruct calcified arteries 
and provide accurate quantitative information. 3D microCT can detect arterial calcification with an intact vascular structure and accurately quantify calcification by the threshold method (93). 3D micro-CT can be combined with histology, immunohistochemistry, and proteomic methods and can be used as a supplementary means of histological examination. It provides a method of obtaining additional information about calcification volume and load from the same artery segment of the same animal (88). Moreover, the increasing practicality and technological development of the 3D Micro CT also provides a new opportunity to visualize and quantify intimal and medial calcification.

\section{Carbon Nanotube-Based Micro-CT}

Carbon nanotube-based (CNT) micro-CT can accurately generate microsecond transmission pulses and control transmission rays and provide higher temporal and spatial resolution. Compared with traditional micro-CT, the sharpness of the CNT calcification image with micro-CT in mouse models increased (94). This is a helpful tool for evaluating vascular calcification in living mice. However, only a few relevant studies have been conducted, and further research is needed to ensure clinical availability.

\section{Combining Micro-CT With 18F-NaF Micro-PET/CT}

${ }^{18} \mathrm{~F}-\mathrm{NaF}$ micro-PET/CT can distinguish between macrocalcification and microcalcification (95). ${ }^{18} \mathrm{~F}-\mathrm{NaF}$ can bind to the calcified surface of small blood vessels (96). It is an essential method in measuring the calcified surface area and metabolic activity degree of calcified plaque according to the uptake of ${ }^{18} \mathrm{~F}-\mathrm{NaF}$ (97). This is an area where micro-CT does not perform well. Therefore, combining micro-CT with ${ }^{18} \mathrm{~F}-\mathrm{NaF}$ micro-PET/CT may become an excellent method to detect vascular calcification. However, its use may be inhibited by the expensive costs and the lack of standard analysis protocols in its clinical application (98).

\section{Nano-CT}

The spatial resolution of nano-CT can be as high as $400 \mathrm{~nm}$, which exceeds micro-CT and can be imaged in the submicron range (90). With nano-CT imaging, the non-calcified groove of a single plaque cell can be detected, and its histopathological correlation corresponds to chondrocyte-like cells. Currently, the possibility of using nano-CT in the body is remote, as it requires inhibiting human physiological activity. Hence, the technology is likely to be used as an imaging method for in vitro analysis (90).

\section{Synchrotron Radiation CT}

The essence of a synchrotron is a circulating particle accelerator, and the X-rays generated by the synchrotron can be used to create $3 \mathrm{D}$ images with resolution up to $1 \mu \mathrm{m}$ (99). Synchrotron radiation $\mathrm{CT}$ is an imaging technology belonging to phasecontrast computed tomography (PCCT), combining phase contrast with micron resolution, promoting superior spatial resolution. Differential phase-contrast imaging, a newly developed synchrotron imaging technology, allows the evaluation of large structures and microscopic details of mouse atherosclerotic plaques and is a detailed three-dimensional morphological evaluation. The three-dimensional characteristics of imaging technology also allow detailed evaluation from different angles (100).

PCCT has high spatial resolution and soft-tissue contrast, which can accurately estimate the constituent and shape of plaque, and reliably classify it, and the results are consistent with histopathology (101). Some studies have shown that (102) phase-contrast CT can accurately identify lipid-rich, fibrous, or calcified plaques and has high diagnostic accuracy (sensitivity $\geq 0.95$; specificity $\geq 0.94$ ). Pfeiffer et al. (103) used phase-contrast CT to examine coronary arteries. The images showed densely calcified plaques and various narrow areas, which were difficult to identify with conventional CT examination (104). However, this technology has not been applied in humans, only in vitro or animal research (105).

Future micro-CT studies are needed if its use can be extended to clinical practice. Suppose micro-CT can suitably integrate three-dimensional data of vascular calcification with a manageable and analyzable platform. In that case, it can be more widely applied and is expected to become a conventional method for vascular calcification analysis in the future.

\section{Ultra-High Resolution CT}

The recently developed ultra-high resolution CT (106) allows images with a slice thickness of $0.25 \mathrm{~mm}$, with a higher spatial resolution than traditional $\mathrm{CT}$, and improves the diagnostic accuracy of CCTA in coronary heart disease and the evaluation of coronary stenosis. However, it has the disadvantage of increasing image noise, and the radiation dose is higher than in CT with the latest wide-coverage detector. A recent study shows that (107) material density imaging based on iodine and calcium improves the diagnostic ability of calcified coronary artery disease in patients with a high calcification score. In recent years, subtraction technology has been combined with CCTA. By removing the interference of artifacts caused by calcified plaque and metal stents, subtraction CCTA can improve the accuracy and efficiency of assessing the stenosis of diseased coronary artery segments (108). Recently, pieces of literature have reported that compared with traditional CCTA, subtraction CCTA can significantly improve the imaging quality of coronary artery calcification.

3D virtual intravascular endoscopy is a less invasive tool, which can be used to analyze the morphology of calcified coronary plaque and improve the assessment of coronary stenosis by CCTA (109).

\section{CONCLUSION AND PROSPECT}

With the development of science and society, imaging inspection equipment, such as CT technology, has been continuously developing since its advent. Different types of CT have their advantages and disadvantages in evaluating vascular calcification (Table 2). Vascular calcification is related to many diseases which seriously affect human health and life. Therefore, early detection and treatment of calcification are of great 
TABLE 2 | Comparison of different CT technologies.

\begin{tabular}{|c|c|c|c|c|}
\hline CT type & Advantages & Disadvantages & Macrocalcification & Microcalcification \\
\hline Dual energy CT & $\begin{array}{l}\text { Ability to eliminate artifacts } \\
\text { Reduce radiation dose (limited) } \\
\text { Improve image quality and contrast } \\
\text { Less contrast dose } \\
\text { Simplify the workflow }\end{array}$ & $\begin{array}{l}\text { Assess poor vascular integrity } \\
\text { Proof of VNC images in calcification } \\
\text { less evidence of role }\end{array}$ & YES & NO \\
\hline Multi-layer spiral CT & $\begin{array}{l}\text { Some post-processing technologies } \\
\text { Non-invasive; Simple; fast high } \\
\text { diagnostic accuracy }\end{array}$ & $\begin{array}{l}\text { Radiation exposure } \\
\text { Increasing odds of associated cancer }\end{array}$ & YES & $\mathrm{NO}$ \\
\hline Micro-CT & $\begin{array}{l}\text { High spatial resolution } \\
\text { Microcalcification can be detected }\end{array}$ & $\begin{array}{l}\text { Long acquisition time } \\
\text { Need deep anesthesia } \\
\text { Poor contrast of the soft tissue } \\
\text { High radiation dose } \\
\text { Not in clinical }\end{array}$ & YES & YES \\
\hline
\end{tabular}

significance. KDIGO experts noted that any patient with vascular calcification that might affect treatment decisions might require an assessment of vascular calcification (45). Increasingly perfect $\mathrm{CT}$ examination makes the diagnostic information captured from images by doctors more accurate and convenient. Also, the evaluation technology of vascular calcification is gradually improving. In the future, it is hoped that CT technology will continue to develop and eventually combine high-definition and low radiation exposure. This will lead to a more extensive application and consequently bring new hope to more patients.

\section{REFERENCES}

1. Barrett H, O'Keeffe M, Kavanagh E, Walsh M, O'Connor EM. Is matrix gla protein associated with vascular calcification? A systematic review. Nutrients. (2018) 10:415. doi: 10.3390/nu10040415

2. Ornellas NP, Joalbo A, Henry M. Coronary artery calcium score: current status. Radiol Brasil. (2017) 50:182-9. doi: 10.1590/0100-3984.2015.0235

3. Agatston A, Janowitz W, Hildner FJ. Quantification of coronary artery calcium using ultrafast computed tomography. J Am Coll Cardiol. (1990) 15:827-32. doi: 10.1016/0735-1097(90)90282-T

4. Becker CR, Knez A, Ohnesorge B, Schoepf UJ, Flohr T, Bruening R, et al. Visualization and quantification of coronary calcifications with electron beam and spiral computed tomography. Eur Radiol. (2000) 10:62935. doi: 10.1007/s003300050975

5. Davis B, Marin D, Hurwitz LM, Ronald J, Ellis MJ, Ravindra KV, et al. Application of a novel CT-based iliac artery calcification scoring system for predicting renal transplant outcomes. Ajr Am J Roentgenol. (2016) 206:436. doi: 10.2214/AJR.15.14794

6. lluri K, Joshi PH, Henry TS, Blumenthal RS, Nasir K, Blaha MJ. Scoring of coronary artery calcium scans: history, assumptions, current limitations, and future directions. Atherosclerosis. (2015) 239:109-17. doi: 10.1016/j.atherosclerosis.2014.12.040

7. Raggi P, O’Neill WC. Imaging for vascular calcification. Semin Dial. (2017) 30:347-52. doi: 10.1111/sdi.12596

8. Smith ER, Hewitson TD, Holt SG. Diagnostic tests for vascular calcification. Adv Chronic Kidney Dis. (2019) 26:445-63. doi: 10.1053/j.ackd.2019.07.001

9. Wilson PW, Kauppila LI, O’Donnell CJ, Kiel DP, Hannan M, Polak $\mathrm{JM}$, et al. Abdominal aortic calcific deposits are an important predictor of vascular morbidity and mortality. Circulation. (2001) 103:152934. doi: 10.1161/01.CIR.103.11.1529

\section{AUTHOR CONTRIBUTIONS}

ZW and LZ conceived the topic and wrote the first draft. LL, GF, $\mathrm{TF}$, and $\mathrm{HJ}$ went through the manuscript and tables. All authors contributed to the article and approved the submitted version.

\section{FUNDING}

This work was supported as follows the National Natural Science Foundation of China (82070455 and 81770450) the Projects from Social Development of Zhenjiang (SH2019087 and SH2018030).

10. Wayhs R, Zelinger A, Raggi P. High coronary artery calcium scores pose an extremely elevated risk for hard events. J Am Coll Cardiol. (2002) 39:22530. doi: 10.1016/S0735-1097(01)01737-5

11. Genders TS, Pugliese F, Mollet NR, Meijboom WB, Weustink AC, van Mieghem CA, et al. Incremental value of the CT coronary calcium score for the prediction of coronary artery disease. Eur Radiol. (2010) 20:233140. doi: 10.1007/s00330-010-1802-y

12. Asafu Adjaye Frimpong G, Owusu IK, Anyitey-Kokor IC, Wiafe-Kwakye C, Aboagye E, Coleman NE, et al. Age-gender distribution of coronary artery calcium score in a black African population in Ghana. Vasc Health Risk Manag. (2018) 14:75-80. doi: 10.2147/VHRM.S160669

13. Rennenberg RJ, Kessels AG, Schurgers LJ, van Engelshoven JM, de Leeuw PW, Kroon AA. Vascular calcifications as a marker of increased cardiovascular risk: a meta-analysis. Vasc Health Risk Manag. (2009) 5:18597. doi: 10.2147/VHRM.S4822

14. Mori H, Torii S, Kutyna M, Sakamoto A, Finn AV, Virmani R. Coronary artery calcification and its progression: what does it really mean? JACC Cardiovasc Imag. (2018) 11:127-42. doi: 10.1016/j.jcmg.2017.10.012

15. Sarwar A, Shaw LJ, Shapiro MD, Blankstein R, Hoffmann U, Cury RC, et al. Diagnostic and prognostic value of absence of coronary artery calcification. JACC Cardiovasc Imag. (2009) 2:675-88. doi: 10.1016/j.jcmg.2008.12.031

16. Rajiah P, Abbara S. CT coronary imaging-a fast evolving world. Qjm. (2018) 111:595-604. doi: 10.1093/qjmed/hcx175

17. Polonsky TS, McClelland RL, Jorgensen NW, Bild DE, Burke GL, Guerci AD, et al. Coronary artery calcium score and risk classification for coronary heart disease prediction. JAMA. (2010) 303:1610-6. doi: 10.1001/jama.2010.461

18. Grundy SM, Stone NJ. 2018 American heart association/American college of cardiology/multisociety guideline on the management of blood cholesterol-secondary prevention. JAMA Cardiol. (2019) 4:58991. doi: 10.1001/jamacardio.2019.0911 
19. Han D, Ji HL, Hartaigh B, Min JK. Role of computed tomography screening for detection of coronary artery disease. Clin Imag. (2016) 40:30710. doi: 10.1016/j.clinimag.2015.07.002

20. Dewey M. Coronary CT versus MR angiography: pro CT-the role of CT angiography. Radiology. (2011) 258:329-39. doi: 10.1148/radiol.10100161

21. Ngam PI, Ong CC, Chai P, Wong SS, Liang CR, Teo LLS. Computed tomography coronary angiography - past, present and future. Singapore Med J. (2020) 61:109-15. doi: 10.11622/smedj.2020028

22. Eckert J, Schmidt M, Magedanz A, Voigtlnder T, Schmermund A. Coronary CT angiography in managing atherosclerosis. Int J Mol Sci. (2015) 16:374056. doi: 10.3390/ijms16023740

23. Lee SE, Villines TC, Chang HJ. Should CT replace IVUS for evaluation of CAD in large-scale clinical trials: effects of medical therapy on atherosclerotic plaque. J Cardiovasc Comp Tomog. (2019) 13:248-53. doi: 10.1016/j.jcct. 2019.06.017

24. Rn A, Sm B, Jl C, Mjb D. How accurate is atherosclerosis imaging by coronary computed tomography angiography? Cardiovasc Comp Tomog. (2019) 13:254-60. doi: 10.1016/j.jcct. 2019.06.005

25. Amanuma M, Kondo T, Sano T, Sekine T, Takase S. Subtraction coronary computed tomography in patients with severe calcification. Int J Cardiovasc Imag. (2015) 31:1635-42. doi: 10.1007/s10554-015-0746-3

26. Park MJ, Jung JI, Choi YS, Ann SH, Youn HJ, Jeon GN, et al. Coronary CT angiography in patients with high calcium score: evaluation of plaque characteristics and diagnostic accuracy. Int J Cardiovasc Imag. (2011) 27(Suppl. 1):43-51. doi: 10.1007/s10554-011-9970-7

27. Skinner JS, Smeeth L, Kendall JM, Adams PC, Timmis A. NICE guidance. Chest pain of recent onset: assessment and diagnosis of recent onset chest pain or discomfort of suspected cardiac origin. Heart. (2010) 96:9748. doi: 10.1136/hrt.2009.190066

28. Zheng M, Wei M, Wen D, Zhao H, Liu Y, Li J, et al. Transluminal attenuation gradient in coronary computed tomography angiography for determining stenosis severity of calcified coronary artery: a primary study with dualsource CT. Eur Radiol. (2015) 25:1219-28. doi: 10.1007/s00330-014-3519-9

29. Ping L, Lei X, Lin Y, Rui W, Jiang H, Sun Z, et al. Blooming artifact reduction in coronary artery calcification by a new de-blooming algorithm: initial study. Sci Rep. (2018) 8:6945. doi: 10.1038/s41598-018-25352-5

30. Osch JV, Mouden M, Dalen JV, Timmer JR, Reiffers S, Knollema $\mathrm{S}$, et al. Influence of iterative image reconstruction on CT-based calcium score measurements. Int J Cardiovasc Imag. (2014) 30:961-7. doi: 10.1007/s10554-014-0409-9

31. Sun Z, Ng C, Xu L, Fan Z, Lei J. Coronary CT angiography in heavily calcified coronary arteries: improvement of coronary lumen visualization and coronary stenosis assessment with image postprocessing methods. Medicine. (2015) 94:e2148. doi: 10.1097/MD.0000000000002148

32. Fuchs A, Kühl JT, Chen MY, Viladés Medel D, Alomar X, Shanbhag SM, et al. Subtraction CT angiography improves evaluation of significant coronary artery disease in patients with severe calcifications or stents-the C-Sub 320 multicenter trial. Eur Radiol. (2018) 28:4077-85. doi: 10.1007/s00330-018-5418-y

33. Criqui $\mathrm{MH}$, Denenberg JO, Ix JH, M Cc Lelland RL, Wassel CL, Rifkin DE, et al. Calcium density of coronary artery plaque and risk of incident cardiovascular events. JAMA. (2015) 311:271-8. doi: 10.1001/jama.2013.282535

34. Schoenhagen P, Ziada KM, Kapadia SR, Crowe TD, Nissen SE, Tuzcu EM. Extent and direction of arterial remodeling in stable versus unstable coronary syndromes : an intravascular ultrasound study. Circulation. (2000) 101:598-603. doi: 10.1161/01.CIR.101.6.598

35. Shemesh J, Apter S, Itzchak Y, Motro M. Coronary calcification compared in patients with acute versus in those with chronic coronary events by using dual-sector spiral CT. Radiology. (2003) 226:4838. doi: 10.1148/radiol.2262011903

36. Jinnouchi H, Sato Y, Sakamoto A, Cornelissen A, Mori M, Kawakami $\mathrm{R}$, et al. Calcium deposition within coronary atherosclerotic lesion: implications for plaque stability. Atherosclerosis. (2020) 306:85-95. doi: 10.1016/j.atherosclerosis.2020.05.017

37. Nakahara T, Dweck MR, Narula N, Pisapia D, Narula J, Strauss HW. Coronary artery calcification: from mechanism to molecular imaging. JACC Cardiovasc Imag. (2017) 10:582-93. doi: 10.1016/j.jcmg 2017.03.005

38. Bailey G, Meadows J, Morrison AR. Imaging atherosclerotic plaque calcification: translating biology. Curr Atheroscler Rep. (2016) 18:51. doi: 10.1007/s11883-016-0601-6

39. McClelland RL. Distribution of coronary artery calcium by race, gender, and age: results from the multi-ethnic study of atherosclerosis (MESA). Circulation. (2006) 113:30-7. doi: 10.1161/CIRCULATIONAHA.105.580696

40. Waltz J, Kocher M, Kahn J, Dirr MK, Burt JR. The future of concurrent automated coronary artery calcium scoring on screening low-dose computed tomography. Cureus. (2020) 12:e8574. doi: 10.7759/cureus.8574

41. Harden SP, Bull RK, Bury RW, Castellano EA, Clayton B, Hamilton $\mathrm{MC}$, et al. The safe practice of CT coronary angiography in adult patients in UK imaging departments. Clin Radiol. (2016) 71:7228. doi: 10.1016/j.crad.2016.04.008

42. Lee SJ, Lee IK, Jeon JH. Vascular calcification-new insights into its mechanism. Int J Mol Sci. (2020) 21:2685. doi: 10.3390/ijms21082685

43. Runge VM, Marquez H, Andreisek G, Valavanis A, Alkadhi H. Recent technological advances in computed tomography and the clinical impact therein. Invest Radiol. (2015) 50:11927. doi: 10.1097/RLI.0000000000000125

44. Shamsul S, Sabarudin A, Hamid HA, Bakar NA, Karim M. Evaluation of radiation dose from coronary CT angiography (CCTA) examination associated with prospective ECG-triggering technique in multidetector 640-slice scanner. J Phys Confer Series. (2020) 1505:012073. doi: 10.1088/1742-6596/1505/1/012073

45. Karohl C, D’Marco Gascón L, Raggi P. Noninvasive imaging for assessment of calcification in chronic kidney disease. Nat Rev Nephrol. (2011) 7:56777. doi: 10.1038/nrneph.2011.110

46. Maurovich-Horvat P, Schlett CL, Alkadhi H, Nakano M, Otsuka F, Stolzmann $\mathrm{P}$, et al. The napkin-ring sign indicates advanced atherosclerotic lesions in coronary CT angiography. JACC Cardiovasc Imag. (2012) 5:124352. doi: 10.1016/j.jcmg.2012.03.019

47. Senoner T, Plank F, Beyer C, Langer C, Birkl K, Steinkohl F, et al. Gender differences in the atherosclerosis profile by coronary CTA in coronary artery calcium score zero patients. J Clin Med. (2021) 10:1220. doi: 10.3390/jcm10061220

48. Leber AW, Knez A, Becker A, Becker C, von Ziegler F, Nikolaou K, et al. Accuracy of multidetector spiral computed tomography in identifying and differentiating the composition of coronary atherosclerotic plaques: a comparative study with intracoronary ultrasound. J Am Coll Cardiol. (2004) 43:1241-7. doi: 10.1016/j.jacc.2003.10.059

49. Nicoll R, Henein MY. Arterial calcification: friend or foe? Int J Cardiol. (2013) 167:322-7. doi: 10.1016/j.ijcard.2012.06.110

50. Toia P, La Grutta L, Sollami G, Clemente A, Gagliardo C, Galia M, et al. Technical development in cardiac CT: current standards and future improvements-a narrative review. Cardiovasc Diagn Ther. (2020) 10:201835. doi: 10.21037/cdt-20-527

51. Cellina M, Gibelli D, Martinenghi C, Oliva G, Floridi C. CT angiography of lower extremities from anatomy to traumatic and nontraumatic lesions: a pictorial review. Emerg Radiol. (2020) 27:441-50. doi: 10.1007/s10140-020-01770-9

52. Raggi P, Chertow GM, Torres PU, Csiky B, Naso A, Nossuli K, et al. The ADVANCE study: a randomized study to evaluate the effects of cinacalcet plus low-dose vitamin D on vascular calcification in patients on hemodialysis. Nephrol Dial Transplant. (2011) 26:132739. doi: $10.1093 / \mathrm{ndt} / \mathrm{gfq} 725$

53. Schmidt B, Flohr T. Principles and applications of dual source CT. Physica Medica. (2020) 79:36-46. doi: 10.1016/j.ejmp.2020 10.014

54. Gottumukkala RV, Kalra MK, Tabari A, Otrakji A, Gee MS. Advanced CT techniques for decreasing radiation dose, reducing sedation requirements, and optimizing image quality in children. Radiographics. (2019) 39:70926. doi: 10.1148/rg.2019180082

55. Lee HA, Lee YH, Yoon KH, Bang DH, Park DE. Comparison of virtual unenhanced images derived from dual-energy CT with true unenhanced images in evaluation of gallstone disease. AJR Am J Roentgenol. (2016) 206:74-80. doi: 10.2214/AJR.15.14570 
56. Meyer M, Haubenreisser H, Raupach R, Schmidt B, Lietzmann F, Leidecker $\mathrm{C}$, et al. Initial results of a new generation dual source CT system using only an in-plane comb filter for ultra-high resolution temporal bone imaging. Eur Radiol. (2015) 25:178-85. doi: 10.1007/s00330-014-3406-4

57. Tesche C, De Cecco CN, Vliegenthart R, Albrecht MH, Varga-Szemes A, Duguay TM, et al. Accuracy and radiation dose reduction using low-voltage computed tomography coronary artery calcium scoring with tin filtration. Am J Cardiol. (2017) 119:675-80. doi: 10.1016/j.amjcard.2016.10.051

58. Tesche C, De Cecco CN, Schoepf UJ, Duguay TM, Albrecht MH, De Santis $\mathrm{D}$, et al. CT coronary calcium scoring with tin filtration using iterative beamhardening calcium correction reconstruction - ScienceDirect. Euro J Radiol. (2017) 91:29-34. doi: 10.1016/j.ejrad.2017.03.011

59. Manta C, Meyer M, Géraud C, Roscher M, Goerdt S, Schoenberg SO, et al. Detection of calcified aortic plaques in an apolipoprotein e animal model using a human computed tomography system for ultra-highresolution imaging: a feasibility study. J Thorac Imag. (2019) 34:417. doi: 10.1097/RTI.0000000000000375

60. Linsen P, Coenen A, Lubbers MM, Dijkshoorn M, Nieman K. Computed tomography angiography with a 192-slice dual-source computed tomography system: improvements in image quality and radiation dose. $J$ Clin Imag Sci. (2016) 6:44. doi: 10.4103/2156-7514.192840

61. Rajiah P, Parakh A, Kay F, Baruah D, Shuai L. Update on multienergy CT: physics, principles, and applications. Radiographics. (2020) 40:200038. doi: 10.1148/rg.2020200038

62. Karçaaltincaba M, Aktaş A. Dual-energy CT revisited with multidetector CT: review of principles and clinical applications. Diag Int Radiol. (2011) 17:181-94. doi: 10.4261/1305-3825.DIR.3860-10.0

63. Siegel MJ, Kaza RK, Bolus DN, Boll DT, Berland LL. White paper of the society of computed body tomography and magnetic resonance on dualenergy CT, part 1: technology and terminology. J Comp Assis Tomog. (2016) 40:841. doi: 10.1097/RCT.0000000000000531

64. Goo HW, Goo JM. Dual-energy CT: new horizon in medical imaging. Korean J Radiol. (2017) 18:555-69. doi: 10.3348/kjr.2017.18.4.555

65. Rassouli N, Etesami M, Dhanantwari A, Rajiah P. Detector-based spectral CT with a novel dual-layer technology: principles and applications. Insights Into Imag. (2017) 8:589-98. doi: 10.1007/s13244-0170571-4

66. Machida H, Tanaka I, Fukui R, Shen Y, Ishikawa T, Tate E, et al. Dualenergy spectral CT: various clinical vascular applications. Radiographics. (2016) 36:1215-32. doi: 10.1148/rg.2016150185

67. Albrecht MH, De C, Joseph SU, Adam S, Marwen E, Domenico DS, et al. Dual-energy CT of the heart current and future status. Eur J Radiol. (2018) 105:110-8. doi: 10.1016/j.ejrad.2018.05.028

68. Kay FU. Dual-energy CT and coronary imaging. Cardiovasc Diagn Ther. (2020) 10:1090-107. doi: 10.21037/cdt.2020.04.04

69. Grant KL, Flohr TG, Krauss B, Sedlmair M, Thomas C, Schmidt B. Assessment of an advanced image-based technique to calculate virtual monoenergetic computed tomographic images from a dual-energy examination to improve contrast-to-noise ratio in examinations using iodinated contrast media. Inv Radiol. (2014) 49:586-92. doi: 10.1097/RLI.0000000000000060

70. So A, Jiang H, Narayanan S, Thibault JB, Imai Y, Dutta S, et al. Dual-energy CT and its potential use for quantitative myocardial CT perfusion. J Cardiovasc Comp Tomog. (2012) 6:308-17. doi: 10.1016/j.jcct. 2012.07.002

71. Wortman JR, Sodickson AD. Pearls, pitfalls, and problems in dual-energy computed tomography imaging of the body. Radiol Clin North Am. (2018) 56:625-40. doi: 10.1016/j.rcl.2018.03.007

72. Schwarz F, Nance JW, Ruzsics B, Bastarrika G, Sterzik A, Schoepf UJ. Quantification of coronary artery calcium on the basis of dual-energy coronary CT angiography. Radiology. (2012) 264:700-7. doi: 10.1148/radiol.12112455

73. Chae EJ, Song JW, Seo JB, Krauss B, Jang YM, Song KS. Clinical utility of dual-energy CT in the evaluation of solitary pulmonary nodules: initial experience. Radiology. (2008) 249:671-81. doi: 10.1148/radiol.24 92071956

74. Kumar V, Min JK, He X, Raman SV. Computation of calcium score with dual-energy computed tomography: a phantom study. $J$
Comput Assist Tomogr. (2017) 41:156-8. doi: 10.1097/RCT.00000000 00000480

75. Sodickson AD. Editor Driving CT developments the last mile: case examples of successful and somewhat less successful translations into clinical practice. SPIE Med Imag. (2017) 10:132. doi: 10.1117/12.2260235

76. Danad I, B ÓH, Min JK. Dual-energy computed tomography for detection of coronary artery disease. Expert Rev Cardiovasc Ther. (2015) 13:134556. doi: 10.1586/14779072.2015.1102055

77. Achenbach S, Goroll T, Seltmann M, Pflederer T, Anders K, Ropers D, et al. Detection of coronary artery stenoses by low-dose, prospectively ECGtriggered, high-pitch spiral coronary CT angiography. JACC Cardiovasc Imag. (2011) 4:328-37. doi: 10.1016/j.jcmg.2011.01.012

78. Danad I, Fayad ZA, Willemink MJ, Min JK. New applications of cardiac computed tomography: dual-energy, spectral, and molecular CT imaging. JACC Cardiovasc Imag. (2015) 8:710-23. doi: 10.1016/j.jcmg.2015.03.005

79. Hideyuki S, Shinichiro F, Yosuke K, Hiroyuki D. Feasibility of macrophage plaque imaging using novel ultrasmall superparamagnetic iron oxide in dual energy CT. Eur J Radiol Open. (2018) 5:87-91. doi: 10.1016/j.ejro.2018.05.003

80. Nitta N, Tsuchiya K, Sonoda A, Ota S, Ushio N, Takahashi M, et al. Negatively charged superparamagnetic iron oxide nanoparticles: a new blood-pooling magnetic resonance contrast agent. Japan J Radiol. (2012) 30:832-9. doi: 10.1007/s11604-012-0133-0

81. Wortman JR, Shyu JY, Dileo J, Uyeda JW, Sodickson AD. Dual-energy CT for routine imaging of the abdomen and pelvis: radiation dose and image quality. Emerg Radiol. (2019) 27:45-50. doi: 10.1007/s10140-019-01733-9

82. Duan X, Ananthakrishnan L, Guild JB, Xi Y, Rajiah P. Radiation doses and image quality of abdominal CT scans at different patient sizes using spectral detector CT scanner: a phantom and clinical study. Abdom Radiol. (2020) 45:3361-368. doi: 10.1007/s00261-019-02247-1

83. Santis D, Domenico, Nam K, Schoepf, Joseph U, Grant, et al. Heavily calcified coronary arteries advanced calcium subtraction improves luminal visualization and diagnostic confidence in dual-energy coronary computed tomography angiography. Invest Radiol. (2018) 53:103-9. doi: 10.1097/RLI.0000000000000416

84. Lell MM, Kramer M, Klotz E, Villablanca P, Ruehm SG. Carotid computed tomography angiography with automated bone suppression: a comparative study between dual energy and bone subtraction techniques. Inv Radiol. (2009) 44:322. doi: 10.1097/RLI.0b013e31819e8ad9

85. Maldonado N, Kellyarnold A, Vengrenyuk Y, Laudier D, Fallon JT, Virmani R, et al. A mechanistic analysis of the role of microcalcifications in atherosclerotic plaque stability: potential implications for plaque rupture. Am J Physiol Heart Circ Physiol. (2012) 303:619-28. doi: 10.1152/ajpheart.00036.2012

86. Danial JSH, Murad F, Saez AG, Moawad MR, Urrico GS, Vancheri F, et al. Computed histological quantification of atherosclerotic plaque microcalcifications. Angiology. (2020) 71:916-9. doi: 10.1177/0003319720939466

87. Badea CT, Drangova M, Holdsworth DW, Johnson GA. In vivo small-animal imaging using micro-CT and digital subtraction angiography. Phys Med Biol. (2008) 53:R319-50. doi: 10.1088/0031-9155/53/19/R01

88. Borland SJ, Behnsen J, Ashton N, Francis SE, Canfield AE. X-ray micro-computed tomography: an emerging technology to analyze vascular calcification in animal models. Int J Mol Sci. (2020) 21:4538. doi: 10.3390/ijms21124538

89. Bos D, Ikram MA, Elias-Smale SE, Krestin GP, Hofman A, Witteman JC, et al. Calcification in major vessel beds relates to vascular brain disease. Arterioscl Thromb Vasc Biol. (2011) 31:2331-7. doi: 10.1161/ATVBAHA.111. 232728

90. Kampschulte M, Langheinirch AC, Sender J, Litzlbauer HD, Krombach GA. Nano-computed tomography: technique and applications. RöFo Fortschritte Gebiet. (2016) 188:146-54. doi: 10.1055/s-0041-106541

91. Vrigneaud JM, Courteau A, Ranouil J, Morgand L, Raguin O, Walker $\mathrm{P}$, et al. Application of the optically stimulated luminescence (OSL) technique for mouse dosimetry in micro-CT imaging. Med Phys. (2013) 40:122102. doi: 10.1118/1.4829499

92. Tamaoki H, Yoshimachi F, Sakurai T, Shin T, Ikari Y. Simultaneous computed tomographic angiography of the coronary and radial arteries. Cardiovasc Int Ther. (2020) 35:185-93. doi: 10.1007/s12928-019-00598-6 
93. Maldonado N, Kelly-Arnold A, Laudier D, Weinbaum S, Cardoso L. Imaging and analysis of microcalcifications and lipid/necrotic core calcification in fibrous cap atheroma. Int J Cardiovasc Imag. (2015) 31:107987. doi: 10.1007/s10554-015-0650-x

94. Wait J, Tomita H, Burk LM, Lu J, Zhou OZ, Maeda N, et al. Detection of aortic arch calcification in apolipoprotein E-null mice using carbon nanotube-based micro-CT system. J Am Heart Assoc Cardiovasc Cerebrovasc Dis. (2013) 2:e003358. doi: 10.1161/JAHA.112. 003358

95. Vgberg W, Persson J, Szekely L, Hertz HM. Cellular-resolution 3D virtual histology of human coronary arteries using x-ray phase tomography. Sci Rep. (2018) 8:11014. doi: 10.1038/s41598-018-29344-3

96. Hsu JJ, Fong F, Patel R, Qiao R, Lo K, Soundia A, et al. Changes in microarchitecture of atherosclerotic calcification assessed by (18)F-NaF PET and CT after a progressive exercise regimen in hyperlipidemic mice. J Nucl Cardiol. (2020). doi: 10.1007/s12350-019-02004-3. [Epub ahead of print].

97. Demer LL, Yin T. Interactive and multifactorial mechanisms of calcific vascular and valvular disease. Trends Endocrinol Metab. (2019) 30:64657. doi: 10.1016/j.tem.2019.06.001

98. Andrews J, Psaltis PJ, Bartolo BAD, Nicholls SJ, Puri R. Coronary arterial calcification: a review of mechanisms, promoters and imaging. Trends Cardiovasc Med. (2018) 28:491-501. doi: 10.1016/j.tcm.2018.04.007

99. Heinzer S, Krucker T, Stampanoni M, Abela R, Meyer EP, Schuler A, et al. Hierarchical microimaging for multiscale analysis of large vascular networks. Neuroimage. (2006) 32:626-36. doi: 10.1016/j.neuroimage. 2006.03.043

100. Bonanno G, Coppo S, Modregger P, Pellegrin M, Stuber A, Stampanoni $\mathrm{M}$, et al. Ultra-high-resolution 3D imaging of atherosclerosis in mice with synchrotron differential phase contrast: a proof of concept study. Sci Rep. (2015) 5:11980. doi: 10.1038/srep11980

101. Hetterich H, Webber N, Willner M, Herzen J, Birnbacher L, Hipp A, et al. AHA classification of coronary and carotid atherosclerotic plaques by grating-based phase-contrast computed tomography. Eur Radiol. (2016) 26:3223-33. doi: 10.1007/s00330-015-4143-z

102. Habbel C, Hetterich H, Willner M, Herzen J, Steigerwald K, Auweter S, et al. Ex Vivo assessment of coronary atherosclerotic plaque by grating-based phase-contrast computed tomography: correlation with optical coherence tomography. Invest Radiol. (2017) 52:223-31. doi: 10.1097/RLI.0000000000000346

103. Pfeiffer F, Reiser M, Rummeny E. [X-ray Phase Contrast: Principles, potential and advances in clinical translation]. Der Radiol. (2018) 58:218-25. doi: 10.1007/s00117-018-0357-9
104. Hetterich H, Willner M, Habbel C, Herzen J, Hoffmann VS, Fill S, et al. Xray phase-contrast computed tomography of human coronary arteries. Invest Radiol. (2015) 50:686-94. doi: 10.1097/RLI.0000000000000169

105. Kwan AC, Pourmorteza A, Dan S, Bluemke DA, Lima J. Nextgeneration hardware advances in CT: cardiac applications. Radiology. (2020) 298:192791. doi: 10.1148/radiol.2020192791

106. Takagi H, Tanaka R, Nagata K, Ninomiya R, Arakita K, Schuijf JD, et al. Diagnostic performance of coronary CT angiography with ultra-highresolution CT: comparison with invasive coronary angiography. Eur J Radiol. (2018) 101:30. doi: 10.1016/j.ejrad.2018.01.030

107. Andreini D, Pontone G, Mushtaq S, Bertella E, Conte E, Segurini C, et al. Diagnostic accuracy of rapid kilovolt peak-switching dual-energy CT coronary angiography in patients with a high calcium score. JACC Cardiovasc Imag. (2015) 8:746-8. doi: 10.1016/j.jcmg.2014.10.013

108. Masafumi K, Daisuke U, Yoshinori F, Daisuke S, Seitaro O, Takeshi N, et al. The effect of heart rate on coronary plaque measurements in 320row coronary CT angiography. Int J CardiovascImag. (2018) 34:1977-985. doi: 10.1007/s10554-018-1415-0

109. Xu L, Sun Z. Virtual intravascular endoscopy visualization of calcified coronary plaques: a novel approach of identifying plaque features for more accurate assessment of coronary lumen stenosis. Medicine. (2015) 94:e805. doi: 10.1097/MD.0000000000 000805

Conflict of Interest: The authors declare that the research was conducted in the absence of any commercial or financial relationships that could be construed as a potential conflict of interest.

Publisher's Note: All claims expressed in this article are solely those of the authors and do not necessarily represent those of their affiliated organizations, or those of the publisher, the editors and the reviewers. Any product that may be evaluated in this article, or claim that may be made by its manufacturer, is not guaranteed or endorsed by the publisher.

Copyright (c) 2021 Zhang, Li, Feng, Fan, Jiang and Wang. This is an open-access article distributed under the terms of the Creative Commons Attribution License (CC $B Y)$. The use, distribution or reproduction in other forums is permitted, provided the original author(s) and the copyright owner(s) are credited and that the original publication in this journal is cited, in accordance with accepted academic practice. No use, distribution or reproduction is permitted which does not comply with these terms. 